\section{THE TREATMENT OF DIABETES MELLITUS.}

abstract of Three Lectures given at the London Hospital

\section{$\mathbf{B Y}$}

\section{O. LEYTON, M.D., D.Sc., F.R.C.P.,} PHYSICIAN TO THE LONDON HOSPITAL.

THe diagnosis and treatment of diabetes mellitus advance rapidly. At comparatively short intervals they need recon. sideration. At the present time we recognize that there are some conditions which simulate diabetes mellitus fairly closely and nevertheless may be considered negligible. We also recognize that during the last few years great advances have been made in the original treatment of diabetes mellitus by restricted diet, and that the correct application of presentday methods will arrest the disease in the majority of patients.

No one has been able to frame a satisfactory definition of diabetes mellitus. This is not difficult to understand when we recognize that it, like many other diseases, is a certain deviation from the average and that there is no unanimity as to what is the minimum deviation which is to be termed disease. In order to take an intelligent view on this question an inquiry must be made into the variability of the quantity of dextrose in the urine and in the blood.

Sugar is present in the urine of normal fasting men even when they have not been submitted to any mental or physical strain : the quantity excreted in an lour is in the region of $20 \mathrm{mg}$., and is not recognizable by the commoner tests for sugar. The quantity of sugar in the urine rises after a meal containing bread and other carbohydrate food, but in all probability this is due to the excretion of unusable sugars and not of dextrose. The administration of 20 grams of dextrose to a fasting man does not lead to any increase in the sugar in the urine, but often 50 grams will do so, raising the excretion to $50 \mathrm{mg}$. an hour. This slight increase of sugar in the urine, an increase which is not recognizable with Benedict's ordinary qualitative test, has been termed "glycuresis" in contradistinction to "glycosuria" "This latter term is reserved for the condition. in which a positive result is obtained by one of the ordinary tests for sugar in the urine. The addition of 25 grams of dextrose to every meal may lead to 1.5 grams dextrose being excreted through the kidneys in twenty-four hours. Benedict takes the view that when that quantity is exceeded in the twenty-four hours it is evidence that there is pancreatic insufficiency. His view is far from universal. Some observers state that sugar may be given by the mouth in surch quantities as to cause nausea without glycosuria occurring, whilst Woodyat, Sansum, and Wilder injected 63 grams intravenously for four hours (a total of 252 grams) without inducing glycosuria. There is obviously no consensus of opinion on the quantity of sugar causing an abnormal increase of sugar in the urine, although there is an agreement that glycosuria is no sudden event-all gradations occur, from definite glycosuria down to a urine containing 0.002 per cent. For convenienoe the assump. tion must be made that glycosuria is present when the urine, mixed with ten volumes of Benedict's qualitative test, reduces the copper on being boiled in a. water-bath for six minates.

The normal quantity of sugar in the blood is no more easily defined than the quantity of sugar in the urine. Every observer should record as many details as possible, although it is probable that some factors still unknown have an influence upon the sugar in the blood. Three main methods of estimating blood sugar are in general use:

Modifications of Lewis and Benedict's Test.-This is based upon picric acid precipitating the proteins, and when heated in alkaline solution with dextrose forming picramic acid. The actual estimation is colorimetric.

Folin and $W u^{\prime} s$ Method.-Blood is deproteinized with tungstic acid; the dextrose reduces aikaline copper solution, and the oxide formed produces an intense blue colour with phosphomolybdic cid. The estimation is colorimetric.

Maclean's Method.-Blood is deproteinized with acid sodium sulphate and dialysed iron; a measured quantity of copper solution containing iodide and iodate is added, the mixture boiled and back-titrated with sodium thiosulphate. Starch is used as an indicator.

The last two methods give similar results, rather lower as a rule than that depending upon picric acid, owing perhaps to the purity of the picric acid not being above suspicion.
The last thousand estimations I have carried out have becn with the Folin and Wu method, and $0.2 \mathrm{c.cm}$. of blood has been taken from the finger; these results influevce my judgement, although most of my statements are based upuus the observations of others.

The factors determining the sugar in the blood after the ingestion of dextrose are manifold; amongst them the follorv. ing have been recognized-length of fast previous to administration, emotional condition, rapidity of absorption of dextrose, quantity of dextrose administered, dilution of dextrose.

The quantity of sugar in the blood of the normal fasting man is usually about 0.09 per cent. If 50 grams of dextrose, dissolved in $200 \mathrm{c.cm}$. of water, be given and the blood taken half an hour later, the percentage of sugar is found to vary from 0.13 to 0.22 , but in the majority of cases within nincty minutes it falls below 0.09 per cent. - that is to say, a hypoglycaemia develops. This fall within a definite time and the hypoglycaemia are of greater value in distinguishıng the abnormal from the normal than the actual maximum percentage of blood sugar found.

All people do not absorb dextrose at the same rate, and when the motility of the stomach is poor the absorption may be slow, the rise of sugar in the blood slow, and the fall slow; therefore the shape of the curve may not be conclusive; but as a rule even with slow absorption the curve falls because the storing mechanism when started is more than able to cope with the sugar absorbed.

\section{Definition.}

An attempt at a definition may be made now. Diabeles mellitus is a defect. in the mechanism the function of which is to store and oxidize carbohydrate, and this makes itself evident by the sugar in the blood becoming excessive.

This sugar seems to be somewhat different from normal blood sugar, as Allen concluded from the fact that dextrose injected into diabetics acted as a diuretic, whilst when injected into normal animals it had no such effect. Quite recently it has been shown that the sugar in the blood of a diabetic possesses a different rotatory value from that in the blood of the normal."

The relation between glycosuria and hyperglycaemia pre. sents many problems. It is true that when the sugar in the blood rises above 0.25 per cent. sugar appears in the urine, and this is not difficult to understand ; but sometimes dextrose appears in the urine in comparatively large quantities whilst the sugar in the blood is less than 0.13 per cent. The amount in the blood which just leads to glycosuria is termed the renal : threshold for sugar, and is found to vary greatly, not only in different individuals, but in the same individual at different times after food. Based upon my own observations, I should place the average threshold at 0.19 per cent. - the highest 0.23 per cent. and the lowest 0.075 per cent.; but other observers found the range much greater-John states from 0.33 per cent. down to 0.04 per cent.

The height of the threshold is not due to an inherent property in the kidney. Hamburger slowed many years ago that the sugar threshold of a frog's kidney could be modified by altering the quantity of sodium bicarbonate in the perfusing fluid, whilst Clarke demonstrated that this was not due to any alteration in the shape of the mesh of the semipermeable membrane of the g'omeruli, but to the tubules failing to absorb sugar in certain circumstances. The fall in this porver of absorption of the tubules some hours after a meal suggests that the ingredients of the plasma control the thresliold. Since we have records of many people who have had sugar in their urine without hyperglycaemia for more than twenty years we must conclude that often this condition is negligible. The fact that occasionally a case of glycosuria develops true diabetes mellitus only leads us to the conclusion that the peculiarity does not protect from diabetes mellitus. Perliaps an appropriate simile exists in the fact that alkaptonuria does not protect from carboluria, but makes the diagnosis a little less simple, just as a low threshold complicates the diagnosis of diabetes mellitus.

\section{Diagnosis.}

The differential diagnosis between glycosuria without hyper. glycaemia and diabetes mellitus is as a rule quite simple, but in doubtful cases laborious. It is my duty, however, to emphasize the fact that no case of glycosuria should be con. sidered negligible until the medical man responsible for its treatment is convinced that his patient is not suffering from diabetes mellitus. The restriction of diet does little harm to 
a case of negligible glycosuria, whilst an unlimited diet does incalculable and irremediable damage to a true diabetic.

The method I adopt in doubtful cases is the following: The ratient has a sample of blood taken and empties the bladder. He is then given a known quantity of dextrose, the amount depending upon the likelihood of his having diabetes mellitus-the less the probability the larger the dose. Blood samp'es are taken at intervals of ten minutes and urine samples at intervals of a quarter of an hour for two hours. In the majority of typical cases a striking phenomenon is noted-namely, that the percentage of sugar in the urine rises after the sugar in the blood has started falling. This great delay in the maximum glycosuria seems typical (Chart 1). The hypoglycaemia which follows within ninety

MEAL OF HAM, HOTATOLS, ICE CREAM I HOUR BEFORE 0.

SOGRAMS DEXTrOSE OY MOUTH AT $a$

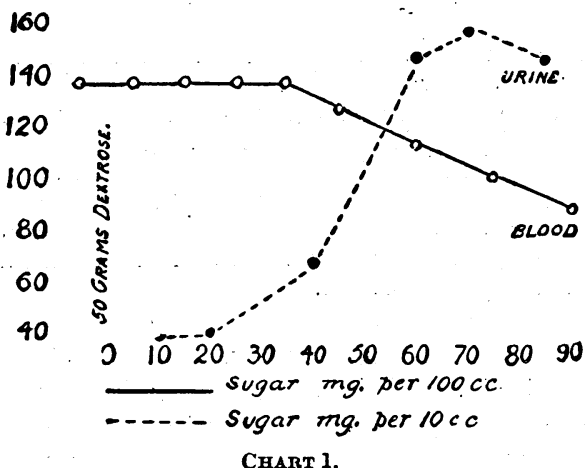

Chart 1.

minutes of the ingestion of the sugar rules out diabetes mellitus (Chart 2). It is the border-line case which is difficult

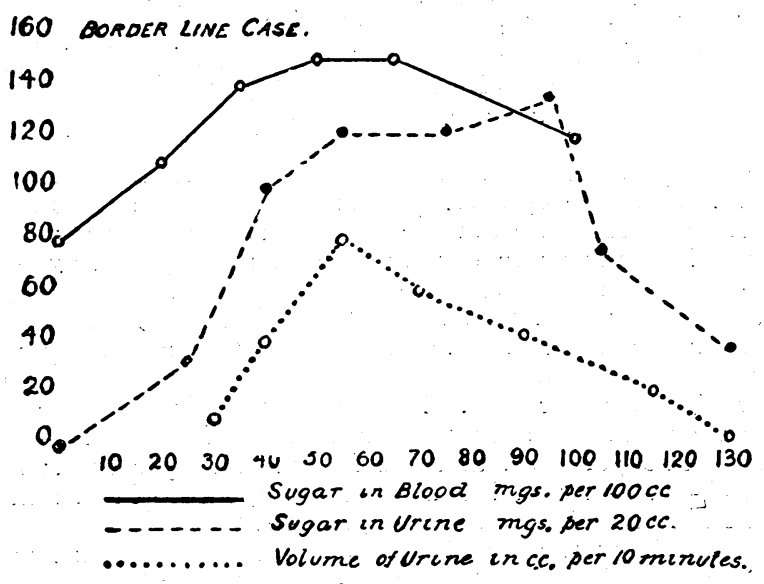

Chart2.

(fall of blood sugar delayed, glycosuria almost coincident with liyperglycaemia, diuresis accompanies hyperglycaemia), and occasionally, as in the case illustrated, the only method of arriving at a conclusion is by leaving the patient upon an ordinary diet and re-examining after as: interval of a few montlis.

Glycosuria with hyperglycaemia arises from other causes than pancreatic inefficiency; it may be due to hyperthyroidism, overactivity of the suprarenals; disease of the pituitary gland, irritation of parts of the central nervous system, and perhaps to certain changes in the liver.

The differential diagnosis may need the ase of all the methads at our disposal. Slight hyperthyroidism rarely leads to glycosuria with hyperglycacmia; considerable overactivity of the thyroid increases the basal metabolism and is usually associated with tachycardia, fine tremor, and a moist slin, three signs which do not arise from pancreatic insuffi. cicncy. Overactivity of the suprarenal glands may arise from anxiety; usually an increased blood pressure can be demonstrated; this when present does not permit true diabetes being excluded. As far as 1 am aware, ketosis and a low respiratory quotient do not accompany glycosuria of Euprarenal origin.

In disease of the pituitary gland the glycosuria is often. intelmittent; the relation letween sugar tolerance and changes in the gland is not easy to understand. In some cases of pituitary tumour the sugar tolerance is low, whilst the patient responds weil to treatment by restricted diet; in others the sugar tolerance is above the normal. A radiograpl of the skull assists diagnosis. The differential diagnosis between pancreatic and hepatic disease may be made by estimating the sugar in the blood after the injection of insulin, or some pancreatic extract which replaces the sub. stance made by the islands of Langerhans. A fall in the sugar indicates that the hyperglycaemia was pancreatic in origin; no distinct change will point to the liver being at fault.

In the past Cammidge has found that the ratio of two substances in the blood-one dextrose, the other a substance which can be hydrolysed into a sugar-is not the same at varying times after food in hepatic and pancreatic dis ease. Space does not permit my giving details. The fact that insulin by itself does not change $a, \beta$ glucose into $\gamma$ glucose without the presence of hepatic extract seems to point towards the possibility of the existence of hepatic glycosuria. ${ }^{2}$

Having arrived at the diagnosis of pancreatic diabetes the patient should be examined with great care to discover any focus of infection. - 'The gums, alveoli, tonsils, antrum, lungs, skin, and urinary tract sliould receive special attention. Any focus should be dealt. with immediately unless a general anaesthetic is an absolute necessity. Local anaesthetics and intraspinal anaesthesia do but little harm, but chloroform, ether, and nitrous oxide should be avoided, because they may, and often do, cause destruction of some: of the cells of the pancreas which are still doing some work. In all probability nitrous oxide with 20 per cent. oxygen would prove of but little barm; but since real relaxation can be obtained only when the mixture is administered under pressure we must wait until a pliilanthropist will supply the fuuds for a theatre which will withstand an extra half-atmosphere pressure.

The treatment of the patient should not be delayed; often every day is of value. The object of the treatment is to reduce the sugar in the blood to 0.10 per cent. without pro: ducing a ketosis or increasing an already existing ketosis; then to find a diet which will not cause the sugar in the blood to rise above 0.15 per cent. at any time.

The only way to arrest the progress of the disease is by ceasing to overwork the pancreas. We have reason to believe that certain forms of glucose in the blood stimulate the pancreas, and if these be present in excess overstimulation which results in progressive delerioration is inevitable. It is true that not every case of diabetes mellitus can be arrested; some are too advanced when they come under treatment; the lives of these must depend upon a supply of insulin. Cases treated correctly may go downhill owing to events against which we do not know how to guard our patients. A patient may have a second or even a third attack of diabetes mellitus; because he has had one or two he is not immune from further. invasions. We are not acquainted with the nature of the toxin which destroys the cells of the islands; the fact that occasionally slight pyiexia accompanies the onset suggests that it may be of microbic origin, but this slight pyrexia is the exception rather than the iule.

The second attack may occur whilst the patient is on a diet which has prevented blood sugar rising above 0.15 per cent. during the previous months or even years; in all respects it simulates the first attack. 'The symptoms are sudden thirst, polyuria, and glycosuria; these are totally different from those occurring in the cases incorrectly treated when tho alteration is insidious, the tolerance gradually diminishing and the glycosuria slowly becoming more frequent.

Another event against which we cannot guard our patients is infection : influenza, pneumonia, whooping-cough, chicken. pox, etc. I have seen all these lead to scich deterioration in the pancreases of arrested cases that upon re-estimating the tolerance of the patient I have found that a decision had to be made between death from inanition or ketosis. It is of interest to note that often in these infections the return of glycosuria occurs before the signs and symptoms of the dise temperature rises. 'This is not difficult to understand when we remember that the injection of a few million dead microbes into a diabetic will cause a temporary hyperglycaemia.

A third erent which may cause advance of the disease is a general anaesthetic, and circumstances may arise which leave no choice - such as naliguant disease anywhere above the fist lumbar segment. 


\section{Treatment.}

The treatment of the patient should depend upon his age, condition, duration of the disease, and severity of the sym. ptoms. For the purpose of describing the treatment patients may be divided roughly into three groups: (1) Recent acute onset. (2) Acnte onset some years ago, or chronic disease of years' standing:- patient emaciated with ketosis. (3) Mild cases, obese but losing weight, often with signs of arteriosclerosis.

Patients belonging to Group 1 should be put to bed and placed upon a beef-tea diet straight away. The risk of ketosis is negligible in quite recent cases. The blood sugar should be estimated after forty-eight hours' fast even if glycosuria persists. I have met at least four cases of true diabetes mellitus with low renal thresholds: glycosuria persisted in these after the blocd sugar was normal $(0.10$ por cent.), and they might have been fasted indefinitely if an attempt had been made to abolish the glycosuria.

If the blood sugar is normal the patient is given 10 grams of carbohydrate in the form of regetables for two days, and c $n$ the third day the blood is taken at intervals after a meal containing 5 grams of carbohydrate given as white bread. If the blood sugar does not at any time rise above 0.14 per cent., the daily diet is raised to carbohydrate 15 grams, with the addition of 15 grams of protein and 15 grams of fat.

After an interval of two more days blood samples are taken following a meal of carbohydrate 10 grams, protein 10 grams, fat 10 grams (carbohydrate given as bread); if satisfactory, the daily diet is raised to carbohydrate, protein, fat, 30 grams of each, and after two days the effect of a meal of 15 grams of each is observed. Provided no hyperglycaemia follows, the diet is raised to carbohydrate, protein, fat, 45 grams of each, for the next two days; then a meal of 20 grams of each is giren, and if that is well borne the daily diet is raised to 60 grams of each.

No advance is then made in carbohydrate or protein, but fat is increased 20 grams daily, until the patient is receiving 25 calories per kilogram body weight, provided no ketosis occurs. This allows a suitable diet to be found. The patient should have the blood examined every few days to see that the blood sugar does not rise above 0.15 per cent., and remain upon 25 calories per kilogram for at least a month. After that time the effect of slight increases in carbohydrate may be observed. Undernutrition for a considerable period is advisable, even if the blood sugar remains low, the rationale being that the maximum recovery of the pancreas will occur if it be given the minimum work with the best blood supply.

Fasting may rest.the pancreas, but a very long fast probably interferes with its nutrition; - we must attempt to steer a course between the Scylla of overwork and the Charybdis of starving the pancreas. The patient may be allowed up after the second week of treatment. Free purging with magnesium sulphate during the first few days of treatment is beneficial to the patient.

If the blood sugar be found to rise above 0.15 per cent. before so liberal a diet be attained an attempt must be made to decide which of the kinds of food is responsib!e. Now and then we come across patients who are able to take more carbohydrate than fat, although the reverse. is the more common. Those who have not met these cases may feel inclined to attribute this to the fact that the energy value of fat is more than donble that of carbohydrate, and that.it is simply due to the general tolerance of the patient being very low; this is, however, not the explanation, because some can take more energy in the form of carbohydrate than in the form of fat.

This fact confirms the view I have taken for some years, that although the general build and main chemical changes in all men are similar, the differences in their metabolism are not less than the differences in their personal appearance.

The emaciated patient with ketosis must have preliminary treatment before fasting. The fat should be removed from his diet immediately, and after two days of very limited protein and carbohydrate fasting should be ordered for two days. If the blood sugar does not fall to normal in that time two days of a diet of carboly ydrate 10 grams, protein 20 grams, should be given, and then the effect of two more fast days observed. Yet a third fast may be tried if tho: second does not accomplish the desired result. In case the third fast proves unsuccessful the patient should be carefully reexamined for some focus of infection; if none be found a meagre diet of carbohydrate 10 grams, protein 30 grams, fat 20 grams, may be given for fourteen days, and then another attempt should be made to reduce the patient's blood to normal. When the blood sugar has been normal for twenty-four hours the effect of food is determined in the same way as in the essay on the recent cases. As a rule the tolerance is found to be very low.

The mild cases need not be fasted: a restricted diet reduces the blood sugar to normal. The obese patients are given very little fat until they have burnt the excess in their bodies. The diet is controlled by frequent estimations of the sugar in the blood.

It is an unfortunate fact that at present the work entailed is considerable, because the time after a meal at which the sugar in the blood rises to the maximum is not constant. Obviously it will depend not only upon the type of carbohydrate given, but also upon what food is given along with the carbolydrate. Samples should be taken every quarter of an hour, beginning half an hour after the meal, for one and a half hours. This need be done only when the final diet is being reached.

Perhaps if the blood sugar can only occasionally be estimated it is wiser to make an attempt to find the diet that wi 1 just allow the threshold being exceeded, and then arrange a permanent diet which is two-thirds that amount. For this purpose the daily examination of the urine for sugar is useless and most misleading. The urine must be passed at intervals after a meal which should contain five-twelfths of the daily diet. Each specimen should be examined separately. Often the twenty-four hour sample will not contain sufficient sugar to give a positive reaction, whilst several separale specimens show that the threshold has been exceeded for a short time after the meal.

Having determined the correct diet, the patient is told to test the urine from time to time, but on no account to increase the diet, even if there be no sugar. The object of the treatment is not to abolish glycosuria but to arrest the disease. Sherrill has recorded cases in which he has observed that patients whose tolerance has deteriorated whilst on a diet which prevented glycosuria maj have the disease arrested by regulating the diet by the sugar conteut of their blood. I, too, have had many similar cases.

\section{Treatment by Insulin.}

How far insulin will modify the treatment of diabetes mellitus is not easily foretold. There is very little doubt that it will prolong the lives of those patients who have only a very small fraction of the islands of Langerhans function. ing. The history of insulin is fairly well known; I regret that space will not permit me to give details, but only to express my admiration of the work of Banting, Macleod, and their fellow workers. The substance termed "insulin." obtained by fractional alcoholic precipitation, seems to be something other than a ferment, and does not give the protein reaction. That it, supplies the substance which the diabetic does not make seems beyond doubt. The diabetio fails to store sugar, as shown by the blood sugar becoming excessive; he fails to burn sugar, as shown by his respiratory quotient being too low. The injection of insulin into a severe diabetic lowers his blood sugar and raises his respiratory quotient.

How it acts needs investigation, but it has been shown that the sugars in the blood of diabetics are not the same as those in the normal individual, and that the abnormal sugars can be converted into the normal by the action of insulin alon' with hepatic extract.

Insulin is not upon the market in Great Britain,* although it is procurable in other countries. The dangers of its use are not, in my opinion, fully realized at the moment. An over. dose may leâd to convulsions, coma, and death; but, since the introduction of a little dextrose acts as a complete and rapid antidote, that dangér seems to be almost negligible, provided that everyone into whose hands insulin finds its way is made acquainted with the facts. In my opinion the danger lies in the administration of limited insulin and un. limited food: The simile between the treatment of diabetes mellitus with insulin and of myxoedema with'thyroid extract is inopportune. No doubt the activity of the thyroid waxes and wanes as necessity arises, and the individual taking thyroid, however carefully the dose be regulated, is not so well as he who has a normally active thyroid. The varia. tions in the activity of the pancreas in all probability aie many hundredfold the variations in the thyroid ; the activity seems to be governed by the sugar in the blood, which in turn depends upon the carbohydrate in the food:

* Since this was written insulin has been placed upon the market and one has had opportunity of confirming the beneficial effects recorded by others. 
Man can live fairly comfortably on 30 grams of carbo. bydrate along with unlimited protein and fat. If a case of severe diabetes be given enough insulin to ensure his utilizing 30 grams of carbohydrate he will escape the symptoms of diabetes mellitus, but his blood sugar will be above 0.15 per cent. during the greater part of the twenty. four hours, which will cause overstimulation of the small amount of pancreas which he still possesses and gradual deterioration. The final stage will be total destruction of the pancreas; then the patient will have to rely for all the sugarburning material on supplies from the outside. In case these fail, life would be extremely short. This is the danger which should be avoided by strictly correlating the diet with the amount of insulin available from the pancreas of the patient together wi:h that injected.

REFERENCEs.
1 Winter and Smith: Journal of Physiol., December, 1922. 2 Forrest, Smith, and Winter: Journal of Physiol., March 23rd.

\section{OBSERVATIONS ON THE INFLUENCE OF INSULIN ON NORMAL METABOLISM IN MAN. BY}

C. H. KELLAWAY, M.D., M.S., M.R.C.P., FOULERTON STUDENT OF THE ROYAL SOCIETY,

DND

T. A. HUGHES, M.B., B.Ch., M.R.C.P., MAJor I.M.S.

(From the Medical Unit, University College Hospital Medical School.)

The study of the respiratory excliange in diabetic patients and in depancreatized dogs by Banting and his co-workers ${ }^{1}$ has shown that the injection of insulin, either alone or com: bined with the administration of sugar by the mouth, causes a pronounced rise in the respiratory quotient. This rise has been generally interpreted as indicating increased con. sumption of glucose, the ability to burn carbohydrate being temporarily restored by the action of insulin. Dudley, Laidlaw, Trevan, and Boock $^{2}$ have recently failed to find any direct evidence of such an action of insulin in normal mice and rabbits, the diminution of circulating sugar being attended by a profound fall in both oxygen consumption and oxtput of carbon dioxide, although they observed in some experiments a marked rise in the respiratory quotient which evidently could not be attributed to increased combustion of glucose.

So far as we are aware, no observations on the influence of insulin on normal metabolism in man have been recorded, and it seemed worth while to record two such experiments which Miss $\mathbf{M} \cdots$, a medical graduate, very kindly allowed us to make upon her. We made three series of observations and on each occasion the subject had no food and only water to drink for the preceding fifteen hours, and during the experiment. She was kept at rest in a bed which was screened off in a corner of a ward. A number of six-minute samples of expired air were collected in a Douglas bag at approximately hourly intervals, some before and some after the injection of insulin. Before the collection of each sample the subject breathed for five minutes through the mouthpiece containing inspiratory and expiratory valves, in order to ensure rcspiratory equilibrium. Obser vations of the pulse and respira. tions were made during this period and during the collection of the sample. A half-hourly record of the rectal temperature was kept throughout the day of each experiment.

The insulin used in these experiments was made under Professor Drummond's direction in the Biochemical Depart. ment at University College, and was tested for us by Dr. 3abkin. We have expressed the dose used in multiples of the amount required to reduce the blood sugar of a rabbit weighing 2 kilos sufficiently to cause convulsions. The injections were made subcutaneously, and chánges in the blood sugar were followed, using Maclean's method for $0.2 \mathrm{c.cm}$. of blood. ${ }^{3}$

A preliminary experiment in which we took a series of eight samples of expired air, four before and four after the injection of two rabbit doses of insulin, gave the subject the necessary training in breathing and furnished an indication of the amount of insulin which could be used in our later experiments. The fall of blood sugar in four hours after this dose of insulin was from 0.123 per cent. to 0.108 per cent., and there were no significant changes in the metabolism. Wo give condensed protocols of our two subsequent series of observations.

In both these experiments there was a marked rise in the respiratory quotient associated with the fall in blood sugar caused by insulin. In Experiment 2 the blood sugar fell from 0.125 per cent. to 0.080 per cent. in one and a quarter hours, and, allowing a quarter of an hour for insulin to be absorbed in sufficient amount to affeot the blood sugar, there was a fall of 0.045 per cent. in an hour. If we assume, with Keith, Rowntree, and Geraghty, ${ }^{4}$ that the blood volume in man is $85 \mathrm{c.cm}$. per kilo of body weight, this represents a loss of $0.045 \times 76 \times 85$ 100

vative estimate of the amount of sugar lost from the blood, because the low level of blood sugar was maintained at 0.080 per cent. for the succeeding two hours, which suggests the attainment of an equilibrium between the sugar disposed of by insulin and that which is in all probability mobilized from the liver during the time of its action.

What is the fate of this sugar lost from the blood? Is it consumed, transformed to glycogen and stored in the liver and muscles, or is it transformed to some other complex, such as fat? If it is stored as glycogen, we are left without any

Experimrnt 2 (Fig. 1). Miss —, ałed 26, weight 76 kilos.

\begin{tabular}{|c|c|c|c|c|c|c|c|c|c|}
\hline Time. & 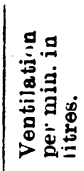 & 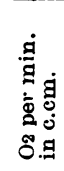 & 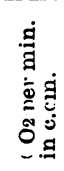 & 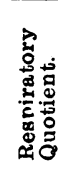 & 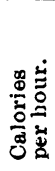 & 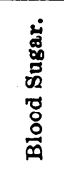 & 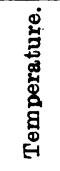 & $\begin{array}{l}\stackrel{\Phi}{\Phi} \\
\dot{\Xi}\end{array}$ & 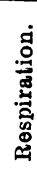 \\
\hline 10 a.m. ... & $4.3 j$ & 230.6 & 174.4 & 0.756 & 65.7 & 0.125 & $99.4^{\circ}$ & 60 & 14 \\
\hline 11.30 & $4: 23$ & 224.0 & 166.0 & 0.741 & 63.5 & - & $99.4^{\circ}$ & $€ 8$ & 15 \\
\hline 12.30 r.m... & Insuli & $-10 \cdot a$ & it dos & injec & & 0.125 & & & \\
\hline 1.45 & 5.34 & $2: 0.5$ & 203.5 & 0.923 & 65.5 & 0.080 & $99.3^{\circ}$ & 74 & 15 \\
\hline 2.45 & 5.17 & 229.0 & 197.0 & 0.860 & 67.0 & 0.183 & $99^{\circ}$ & 71 & 19 \\
\hline 3.30 & 4.75 & 223.3 & 180.0 & 0.806 & 61.2 & 0.080 & - & 68 & 20 \\
\hline
\end{tabular}

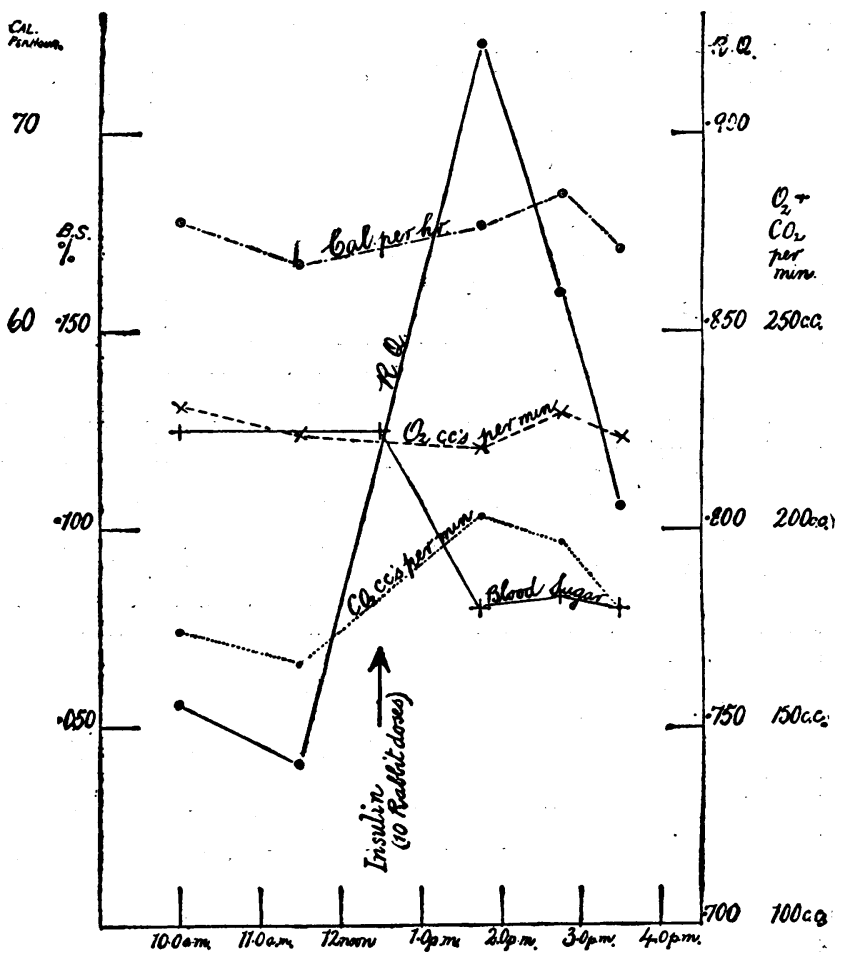

Fig. 1.-The effect of ten rabbit doses of insulin on the metabolism of a normal person weighing 76 kilos. R.Q. = respiratory quotient. Calories per hour calculated from observed oxygen consumption and R.Q. on the assumption that the rise in R.Q. is wholly due to increased combustion of carbohydrate.

At 12.30 ten rabbit doses of insulin were administered. Ther was a definite increase in the ventilation rate one and a quarter hours later, but the patient showed no symptoms till 2.20 p.m when she When she commenced topar lond and that sh that our voices were unduly loud and that she was feeling drowsy. The drowsiness increased, "coming and going in waves," and the change in mental condition became very striking. The blood pressure was normal and there were no neurological signs. Att 4 p.m. 50 grams of glucose were given and the symptoms rapidly disappeared. 